\title{
Functional MRI and Diffusion Tensor Imaging of Brain Reorganization After Experimental Stroke
}

\author{
Rick M. Dijkhuizen • Kajo van der Marel • \\ Willem M. Otte • Erik I. Hoff • Jet P. van der Zijden • \\ Annette van der Toorn • Maurits P. A. van Meer
}

Received: 27 September 2011 / Revised: 23 December 2011 /Accepted: 27 December 2011 /Published online: 24 January 2012

(C) The Author(s) 2012. This article is published with open access at Springerlink.com

\begin{abstract}
The potential of the adult brain to reorganize after ischemic injury is critical for functional recovery and provides a significant target for therapeutic strategies to promote brain repair. Despite the accumulating evidence of brain plasticity, the interaction and significance of morphological and physiological modifications in post-stroke brain tissue remain mostly unclear. Neuroimaging techniques such as functional MRI (fMRI) and diffusion tensor imaging (DTI) enable in vivo assessment of the spatial and temporal pattern of functional and structural changes inside and outside ischemic lesion areas. This can contribute to the elucidation of critical aspects in post-stroke brain remodeling. Task/stimulus-related fMRI, resting-state fMRI, or pharmacological MRI enables direct or indirect measurement of neuronal activation, functional connectivity, or neurotransmitter system responses, respectively. DTI allows estimation of the structural integrity and connectivity of white matter tracts. Together, these MRI methods provide an unprecedented means to (a) measure longitudinal changes in tissue structure and function close by and remote from ischemic
\end{abstract}

R. M. Dijkhuizen $(\bowtie) \cdot$ K. van der Marel $\cdot$ W. M. Otte

J. P. van der Zijden • A. van der Toorn • M. P. A. van Meer

Biomedical MR Imaging and Spectroscopy Group,

Image Sciences Institute, University Medical Center Utrecht,

Utrecht, The Netherlands

e-mail: rick@invivonmr.uu.nl

W. M. Otte

Department of Child Neurology,

Rudolf Magnus Institute of Neuroscience,

University Medical Center Utrecht,

Utrecht, The Netherlands

\section{E. I. Hoff}

Department of Neurology, Maastricht University Medical Center and Atrium Medical Center Heerlen,

Maastricht/Heerlen, The Netherlands lesion areas, (b) evaluate the organizational profile of neural networks after stroke, and (c) identify degenerative and restorative processes that affect post-stroke functional outcome. Besides, the availability of MRI in clinical institutions as well as research laboratories provides an optimal basis for translational research on stroke recovery. This review gives an overview of the current status and perspectives of fMRI and DTI applications to study brain reorganization in experimental stroke models.

Keywords Brain plasticity · Functional connectivity . Diffusion tensor imaging $\cdot$ Functional MRI $\cdot$ Neuronal network $\cdot$ Stroke

\section{Introduction}

Stroke is a major cause of long-term disability throughout the world, leaving more than half of the patients dependent on daily assistance. Nonetheless, most patients exhibit a certain degree of recovery in the weeks, months, and sometimes even years following stroke, which may be directly related to structural and functional modifications in surviving brain tissue. Several experimental and human stroke studies have reported vicarious function of ipsilesional and contralesional brain regions [1-3], which may contribute to restoration of functions, although the exact mechanisms that lead to functional recovery remain largely unclear. Elucidation of the critical pathways in post-stroke recovery would not only provide important fundamental insights in brain function and plasticity, but could also lead the way towards development of new rehabilitation strategies for recovering stroke patients.

Imaging modalities, such as MRI, may significantly contribute to the research on stroke recovery by enabling serial 
in vivo whole-brain measurements of functional and/or anatomical changes in injured brain. Established MRI methods such as $\mathrm{T}_{2-}$, diffusion-, and perfusion-weighted MRI are frequently applied for assessment of acute stroke pathophysiology in clinical diagnosis and preclinical research [4-6], but MRI may also be employed to characterize long-term alterations in brain structure and function after stroke [5-8]. Over the years, various studies have demonstrated that functional MRI (fMRI) can be used to study alterations in cortical and subcortical activation patterns in stroke patients and animal models of stroke [9-11]. In addition, structural changes in gray and white matter areas have been successfully measured with diffusion tensor imaging (DTI) after clinical as well as experimental stroke $[12,13]$. The following paragraphs will give an overview of diverse functional MRI and DTI methods and their application in animal models of stroke.

\section{Task/Stimulation-Related Functional MRI}

Functional MRI methods are traditionally sensitized to changes in cerebral hemodynamics in response to neuronal activity. The most frequently applied fMRI method is blood oxygenation level-dependent (BOLD) gradient-, or spinecho MRI. Alternative techniques include arterial spin labeling (i.e., cerebral blood flow-weighted MRI) and steadystate susceptibility contrast-enhanced MRI in combination with a (super)paramagnetic blood pool agent [i.e., cerebral blood volume (CBV)-weighted MRI]. The principles of these different fMRI methods have been extensively described by Mandeville and Rosen [14] and Harel et al. [15].

Functional MRI enables assessment of human brain activation in relation to sensory, motor, or cognitive tasks or stimuli. Besides, fMRI can also be applied in animal experiments. Despite the fact that anesthesia (type, depth, and duration) may significantly influence neurovascular coupling [16], fMRI in animals has been successfully executed with anesthesia protocols that preserve hemodynamic responsiveness to neuronal activity [17]. Experimental fMRI studies have provided important insights into the physiological basis of fMRI responses, fundamentals of hemodynamic and metabolic aspects of neuronal activation, and consequences of lesions on cerebral activity $[17,18]$, which will be discussed below in relation to stroke injury and recovery.

We and others have applied fMRI in rodent stroke models to assess changes in sensorimotor system activation in response to limb stimulation [19-27]. To maximize the sensitivity and specificity of the detection of activation responses, most of these $\mathrm{fMRI}$ studies have taken advantage of high magnetic field strength ( $\geq 7 \mathrm{~T})[22,23,26]$ and/or a (super)paramagnetic blood pool agent [19-25]. This not only increases the signal-to-noise and/or contrast-to-noise ratio of the fMRI signal, but also reduces the contribution of unspecific signal from large blood vessels [14, 15]. Consistent with fMRI studies in stroke patients, these fMRI experiments in rodent stroke models have revealed diminished activation in the ipsilesional sensorimotor cortex [19-21, 24, 26, 27], enhanced contralesional activation [19-21], and chronic reinstatement of perilesional activity $[20,21$, 26, 27].

Functional MRI-detected activation response to forelimb stimulation may be transiently absent in the representational somatosensory cortex after experimental stroke, despite normal structural appearance on diffusion- and $\mathrm{T}_{2}$-weighted MR images and intact vasoreactivity [20, 21, 26, 27]. Functional denervation, depression, or deterioration of surviving neurons, as well as derangement of neurovascular coupling, could explain the temporary lack or responsiveness. The preservation or reinstatement of activation within the ipsilesional sensorimotor cortex has been found to be associated with functional recovery after transient middle cerebral artery occlusion in rats $[21,26]$, which emphasizes the significance of reorganization of affected cortical representational fields for post-stroke functional recovery.

Some studies have reported a rise in contralesional fMRI activity after experimental unilateral cerebral ischemia [19-21], which correlated with the extent of tissue injury in the ipsilesional sensorimotor cortex [21]. The relevance of enhanced activation in the unaffected hemisphere remains controversial; it may hypothetically contribute to functional recovery, but could also be a direct pathophysiological consequence (e.g., due to broad disinhibition) following stroke.

To assess neuronal signal transmission between the contralesional and ipsilesional sensorimotor cortex after unilateral stroke, we have measured perilesional BOLD signals measured in response to direct intracortical stimulation in the contralesional hemisphere of rat brain [28]. Despite disturbed sensorimotor function in the first days after stroke, BOLD responses were preserved in the perilesional sensorimotor cortex, although the time-to-peak was significantly delayed, possibly due to deficient neurovascular coupling (Fig. 1). These results indicated that interhemispheric functional connectivity is sustained and that-in spite of dysfunction-perilesional cortical tissue can be activated subacutely after unilateral stroke.

Besides assessment of neural correlates of functional deficits and recovery, fMRI may also provide a means to evaluate treatment effects on brain function after stroke. For example, restoration of activation responses to electrical forelimb stimulation has been demonstrated with fMRI in rats treated with a $\mathrm{Ca}^{2+}$ antagonist after permanent MCA occlusion [25]. In studies on the therapeutic efficacy of delayed albumin or lithium administration in a rat stroke model, enhanced fMRI activity was detected in the perilesional somatosensory cortex in albumin- or lithium-treated 

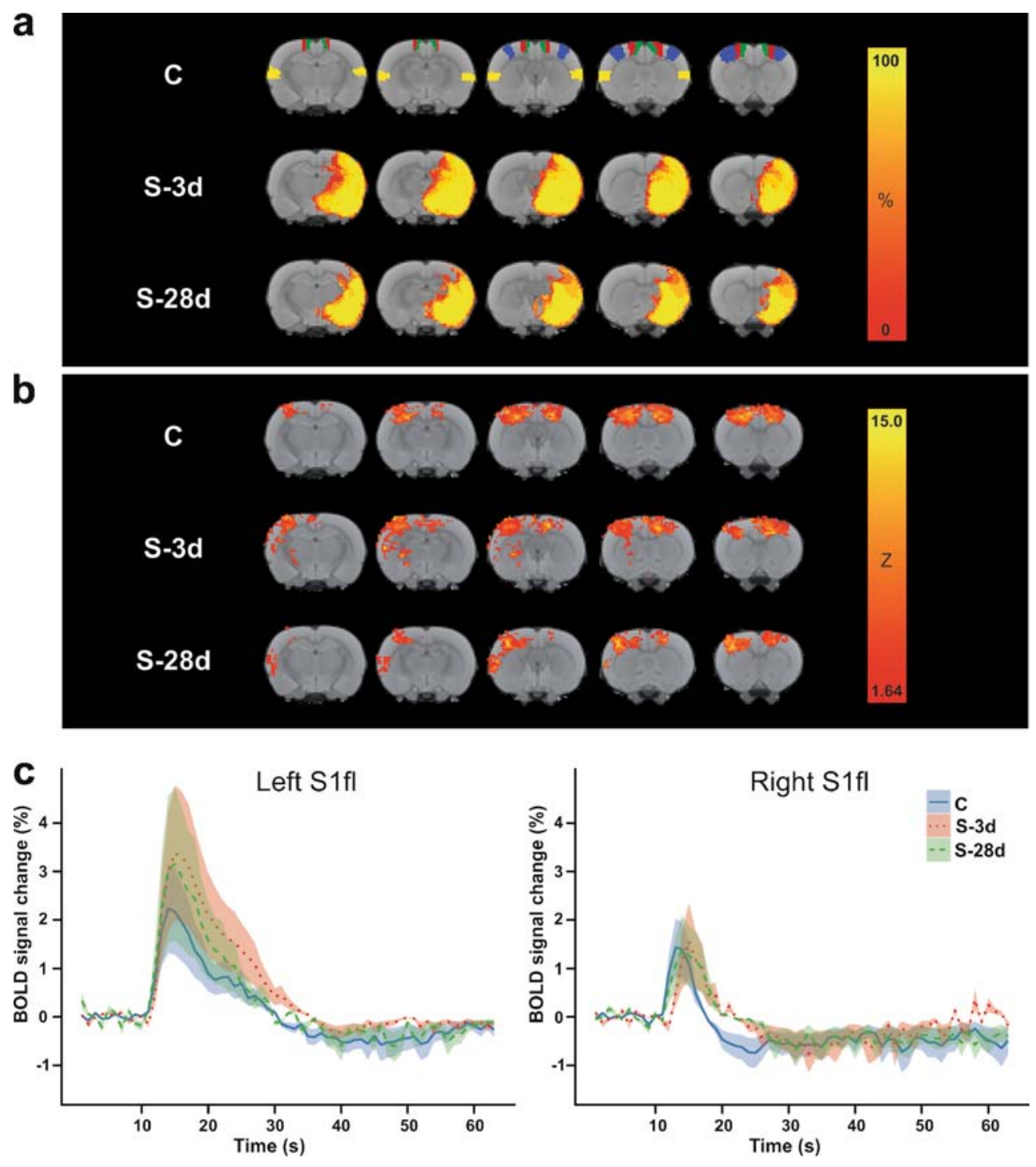

Fig. 1 a Color-coded local incidence of $\mathrm{T}_{2}$-based lesion (percent of group size) in control rats $(C ; n=6)$ and rats at $3(S-3 d ; n=6)$ or 28 days after stroke $(S-28 d ; n=4)$-induced by 90 -min occlusion of the right middle cerebral artery - overlaid on consecutive coronal rat brain slices from a $\mathrm{T}_{2}$-weighted MRI template. Bilateral sensorimotor regions are depicted on the template of group C (green, secondary motor cortex; red, primary motor cortex; blue, forelimb region of the primary somatosensory cortex; yellow, secondary somatosensory cortex). b Mean maps of significant BOLD activation in response to direct intracortical stimulation $(3 \mathrm{~s}$ period of $1.5 \mathrm{~mA}$ with $0.3 \mathrm{~ms}$ duration at $300 \mathrm{~Hz}$ in

animals, while lesion size was not different from salinetreated animals $[22,23]$.

\section{Resting-State Functional MRI}

An alternative fMRI method, known as resting-state fMRI (rs-fMRI), assesses spatial functional correlations within neural networks without the need of a stimulation paradigm [29]. During the last decade, rs-fMRI has been increasingly applied as a tool to study alterations in the brain's intrinsic

$50 \mathrm{~ms}$ trains, repeated five times per second) of the left (contralesional) primary motor cortex in rats from groups $\mathrm{C}, \mathrm{S}-3 \mathrm{~d}$, and S-28d. Colorcoded maps display $Z$ values, overlaid on consecutive coronal rat brain slices from a $\mathrm{T}_{2}$-weighted template. c Averaged BOLD signal time courses (lines) \pm s.e.m. (shadings) in left (contralesional) (left graph) and right (ipsilesional) (right graph) forelimb regions of the primary somatosensory cortex (S1fl) in response to intracortical stimulation of the left (contralesional) primary motor cortex (onset at time $=10 \mathrm{~s}$ ), for groups C (solid blue lines), S-3d (dotted red lines), and S-28d (dashed green lines). From Van Meer et al. [28]

functional architecture as potential physiological correlates of neurological and psychiatric disorders [30-32]. Spontaneous fluctuations in baseline ("resting-state") neuronal signaling are reflected in low-frequency fluctuations $(<0.1 \mathrm{~Hz})$ of the BOLD signal and show temporal coherence between anatomically connected brain regions within a particular neuronal network, such as the sensorimotor system [29, 33, 34]. Throughout the gray matter, the extent of synchronization between these low-frequency BOLD fluctuations is related to functional connectivity. Correlation of these 
signals with electroencephalographic brain activity has indicated that these slow hemodynamic fluctuations are associated with neuronal function [35-37].

Several groups have shown that functional connectivity measurements with rs-fMRI are feasible in anesthetized rats [38-42], which has formed the basis of our recent rs-fMRI studies in rats to depict spatiotemporal characteristics of reorganization in functional neuronal networks after ischemic brain injury $[43,44]$. We found that strong correlations between rs-fMRI signals in ipsilateral and contralateral cortical sensorimotor regions disappear in the first days after unilateral stroke in rats, indicative of loss in interhemispheric functional connectivity [44], which is in correspondence with findings in human patients subacutely after stroke [45]. In subsequent weeks and coinciding with recovery of sensorimotor function, interhemispheric functional connectivity was partially retrieved [44] (see Fig. 2a). On the other hand, intrahemispheric functional connectivity between primary somatosensory and motor cortices was preserved in the lesion borderzone and moderately enhanced contralesionally, which may reflect intact and/or new formations of local neuronal connections. This was supported by additional manganese-enhanced MRI (MEMRI) experiments [43]. MEMRI is an MRI-based in vivo tract tracing method that is based on the detection of neuronal distribution of paramagnetic $\mathrm{Mn}^{2+}, \mathrm{a} \mathrm{Ca}^{2+}$ analog that can enter active neurons through $\mathrm{Ca}^{2+}$ channels [46]. Injection of $\mathrm{Mn}^{2+}$ in the brain has been shown to result in neuronal uptake and transsynaptic transport along connective pathways [47]. In our combined rs-fMRI and MEMRI study, we found that injection of $\mathrm{MnCl}_{2}$ in the contralesional sensorimotor cortex resulted in locally increased uptake, indicative of locally enhanced structural connectivity between neurons, as well as decreased transcallosal transfer, corroborative of the loss of interhemispheric functional connectivity [43]. These findings underline the link between functional and structural reorganization of neuronal networks after stroke.

Functional connectivity has been most often computed by calculation of correlations between the low-frequency BOLD fluctuations in an a priori-selected brain region and lowfrequency BOLD fluctuations from other voxels in the brain. Beside region-based functional connectivity analyses, functional connectivity may also be assessed at a whole network level by means of graph analysis of rs-fMRI data [32, 48, 49]. Networks can be represented as graphs containing nodes and

Fig. 2 a Interhemispheric functional connectivity, b normalized clustering coefficient, $\mathbf{c}$ normalized shortest path length, and $\mathbf{d}$ small-world index of bilateral sensorimotor cortex [calculated from combined bilateral sensorimotor cortical regions (see Fig. 1a)], in rats with large lesions involving both subcortical and cortical tissue $(n=9)$, before stroke (day 0), and at post-stroke days 3, 7, 21, and 70 [44]. Data are shown as mean \pm s.d. $* P<0.05$ vs. day 0 (based on repeated measures linear mixed model analysis and post hoc Tukey testing) a
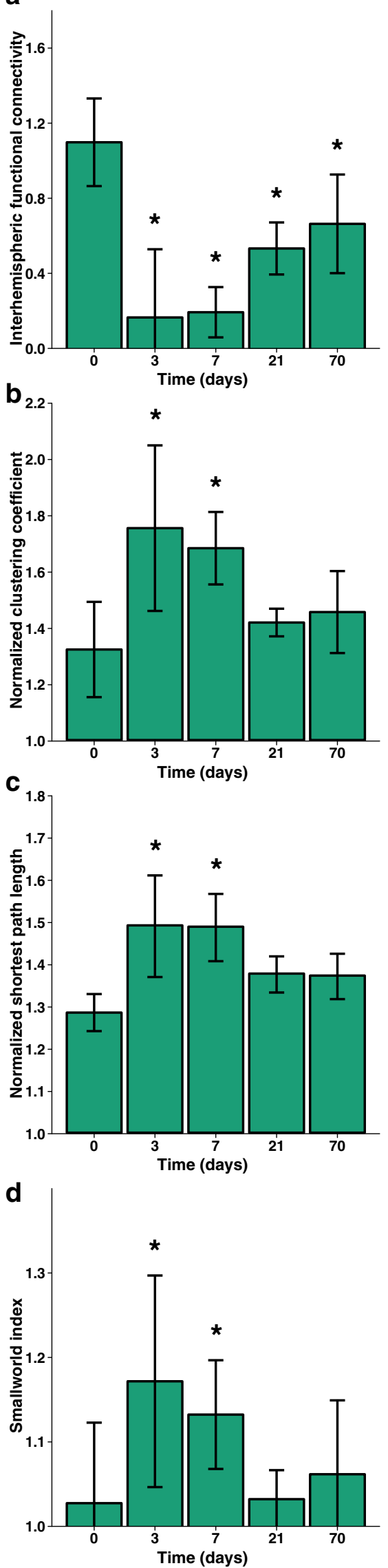
edges. In the case of rs-fMRI, the image voxels represent the nodes and the intervoxel correlations between low-frequency BOLD fluctuations represent the edges. The network's structure can be assessed by measuring its clustering coefficient, a measure of segregation that reflects the degree to which nodes are clustered, and the shortest path length, a measure of integration that reflects the minimum number of edges between any pair of nodes. A high clustering coefficient and low average shortest path length indicate a small-world network topology, which is proposed to be an optimal network configuration for global information transfer and local processing [48-50]. Graph-based analysis of rs-fMRI data from chronic human stroke subjects showed a decrease in clustering coefficient and a shift towards a random network configuration in the motor system [51]. Graph analysis of our rs-fMRI data from rats recovering from unilateral stroke points towards increases in clustering coefficient, average shortest path length, and small-world index in the intact bilateral sensorimotor cortices at early stages, which normalize chronically (Fig. 2b-d) (unpublished observations). This emphasizes that large-scale networks outside the ischemic area rearrange over time. Speculatively, higher values of the calculated network parameters subacutely after stroke may be a sign of diffuse overconnectivity that precedes maturation towards a normally functioning circuitry within the reorganized network.

\section{Pharmacological MRI}

Abovementioned fMRI studies in experimental stroke models are based on measurement of cerebral activity under baseline conditions or in responses to somatosensory stimuli. Alternatively, neuronal activation may be evoked by pharmacological stimulation. Pharmacological MRI (phMRI) is a variant of fMRI that assesses hemodynamic changes associated with cerebral activity in response to centrally acting pharmacological agents [52]. A pioneering phMRI study in rats after stroke has shown a strongly diminished cerebral activation response to injection of the $\mathrm{GABA}_{\mathrm{A}}$ antagonist bicuculline, indicative of functionally compromised brain tissue [53]. To investigate the longterm effect of focal cerebral infarction on the cholinergic system, which has been implicated in post-stroke cognitive impairment, we have carried out phMRI in combination with administration of pilocarpine - a muscarinic cholinergic agonist - to measure the activation of the cholinergic system after photothrombotic infarction in rat cerebral cortex [54]. Hemodynamic responses to cholinergic muscarinic receptor stimulation were significantly reduced inside the ischemic area at 1 and 3 weeks and in areas distant from the lesion at 3 weeks after right-sided frontal cortical infarction, while cholinergic cell number was unchanged. Furthermore, we have observed reduced cholinergic muscarinic receptor responsiveness in structurally preserved somatosensory projection cortex at 2 weeks after subcortical ischemic damage induced by transient occlusion of the right middle cerebral artery in rats (Fig. 3) (unpublished observation). These phMRI data demonstrate significantly altered cholinergic receptor responsiveness distant from focal cerebral ischemic infarction.

\section{Diffusion Tensor Imaging}

The previous sections deal with functional MRI methods that can be employed to identify altered patterns of brain activity after stroke. Changes in functional brain organization, however, are often closely associated with structural modification of neuronal elements in the brain. DTI offers an MRI-based means for the assessment of neuroanatomical changes associated with brain injury and repair. DTI informs on the three-dimensional displacement of tissue water, mathematically characterized by an effective diffusion tensor consisting of nine matrix elements, which can be exploited to assess the microstructure of gray and white matter tissue (see Basser and Jones [55] and Mori and Zhang [56] for reviews). As the diffusion of tissue water is restricted by the presence and orientation of biological barriers such as cell membranes and myelinated fibers, strokeinduced structural modifications therein can significantly alter the characteristics of tissue water diffusion, such as the DTI-derived axial, radial, and mean diffusivity (MD), as well as fractional anisotropy (FA) [57]. This may involve different processes at different post-stroke stages, ranging from acute cell swelling (causing MD decrease) and subacute cell lysis and demyelination (causing MD increase and FA decrease), to chronic axonal regeneration or remyelination and gliosis (causing FA increase) [13, 57, 58].

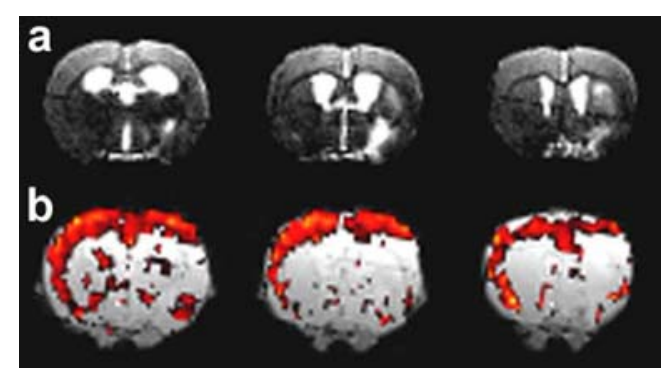

Fig. 3 a $T_{2}$-weighted $M R$ images of consecutive coronal rat brain slices at 14 days after 60 min occlusion of the right middle cerebral artery. The unilateral subcortical ischemic lesion is characterized by increased $\mathrm{T}_{2}$-weighted signal intensity. b Maps of significant positive CBV responses induced by intravenous injection of pilocarpine- a muscarinic cholinergic agonist-as calculated from contrast-enhanced phMRI (see Hoff et al. [54] for methods), overlaid on anatomical MRI images. Muscarinic activation responses were diminished in large part of the right (ispilesional) cortex, despite normal appearance on structural MRI 


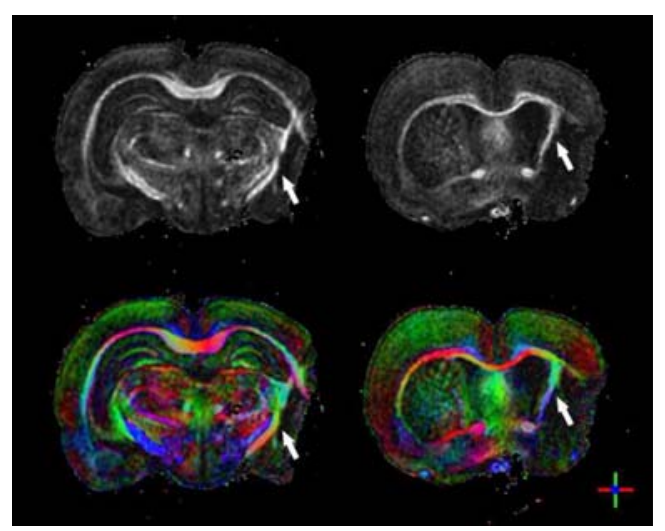

Fig. 4 Ex vivo fractional anisotropy maps [grayscale values (top) and color-coded orientations (bottom)] of consecutive coronal rat brain slices at 11 weeks after 90 min occlusion of the right middle cerebral artery, calculated from DTI with high spatial $\left(200 \times 200 \times 200 \mu^{3}\right.$ voxel size) and angular resolution (120 diffusion-weighted directions). Arrows indicate the increased fractional anisotropy in white matter around the lesion (with low fractional anisotropy)

Since white matter tracts are composed of highly oriented fibers, which cause relatively high anisotropy of diffusing tissue water, DTI is very suitable to measure effects on white matter integrity. Studies in patients and animals have reported loss of FA in ipsilesional white matter subacutely after stroke, which has been linked to demyelination or axonal loss $[6,12,59]$. A few serial DTI studies in experimental stroke models have demonstrated that this initial decrease of FA may be chronically followed by normalization or elevation in the ischemic lesion borderzone [58], which could be further enhanced by treatment with neural progenitor cells [60], sildenafil [61], or erythropoietin [62]. This area revealed a high density of axons and myelin on postmortem histological sections [60,61]. Furthermore, part of the ipsilesional internal capsule with elevated FA in post-stroke rat brains also showed significant manganese enhancement on $T_{1}$-weighted MRI after injection of the paramagnetic neuronal tracer in the perilesional sensorimotor cortex [58]. This suggests that rearrangement of white matter in the ischemic boundary is accompanied by preservation or restoration of neuronal connectivity. Figure 4 shows postmortem high-resolution FA maps that we collected at 11 weeks post-stroke. These data clearly illustrate the FA rise in perilesional white matter at chronic stages after stroke. In accordance with these findings in animal stroke models, elevated FA in ipsilesional corticospinal tracts has also been found in chronic stroke patients, which was associated with improved motor function [63]. Evidently, structural integrity of the corticospinal pathway appears critical for a favorable outcome in sensorimotor performance after stroke.

The DTI-derived diffusion anisotropy and principal diffusion direction in white matter tissue can be used to model the architecture of neuronal fibers, visualized by orientation- based color-coded FA maps (see Fig. 4) or three-dimensional fiber tract maps computed with tractography algorithms [56]. However, the angular resolution of DTI, which determines the extent to which fiber orientation can be estimated, is limited [64]. This can significantly affect the accuracy of tractography. DTI provides a single principal diffusion direction per voxel, which complicates the resolution of complex white matter architecture with crossing, kissing, bending, or fanning fibers within a voxel. In addition, DTI assumes a Gaussian model of diffusion, which may not be appropriate for restricted diffusion in biological tissues [65]. Alternative diffusionimaging schemes, such as q-ball imaging [66], spherical deconvolution [67], and diffusion spectrum imaging [68] have been developed to address these issues of multiple fiber orientations in a single voxel and to improve the quality of fiber tracking. A recently introduced method, diffusion kurtosis imaging, allows quantification of the degree to which tissue water diffusion is non-Gaussian [65]. This enables further characterization of the complexity of tissue microstructure in gray and white matter by accounting for the diffusion kurtosis as a result of the present cellular compartments and membranes $[69,70]$. These advanced diffusion imaging techniques, however, require high hardware standards and long scan durations, as well as special analysis software. Currently, this impedes widespread application in preclinical and clinical settings.

\section{Conclusion}

MRI offers a powerful means to assess functional activity and structural integrity of the brain, which can be exploited to evaluate the spatiotemporal pattern of changes after stroke in both clinical and preclinical settings. Especially the combination of in vivo functional MRI and DTI techniques provides a unique complementary approach to investigate the interaction of reorganization of neuronal networks in relation to function. MRI may therefore significantly contribute to (a) elucidation of cerebral rearrangements that underlie functional recovery, (b) prediction of outcome, and (c) monitoring of therapeutic strategies that promote brain repair. In the coming years, multiparametric MRI studies aimed at mapping the complex process of brain reorganization after ischemic injury, conducted in parallel in human patients and (transgenic) animal models, may help to unravel the mechanisms that underlie loss and restoration of function after stroke. Ultimately, this could lead to the development of more effective diagnosis and treatment strategies for recovering stroke patients.

Acknowledgments Part of the presented work was funded by the Alexandre Suerman program of the University Medical Center Utrecht, Utrecht University's High Potential program, and the European Union's Seventh Framework Programme (FP7/2007-2013) under grant agreement no. 201024 and no. 202213 (European Stroke Network). 
Open Access This article is distributed under the terms of the Creative Commons Attribution Noncommercial License which permits any noncommercial use, distribution, and reproduction in any medium, provided the original author(s) and source are credited.

\section{References}

1. Dancause N. Vicarious function of remote cortex following stroke: recent evidence from human and animal studies. Neuroscientist. 2006;12(6):489-99.

2. Nudo RJ. Postinfarct cortical plasticity and behavioral recovery. Stroke. 2007;38(2 Suppl):840-5.

3. Ward NS. Neural plasticity and recovery of function. Prog Brain Res. 2005;150:527-35.

4. Baird AE, Warach S. Magnetic resonance imaging of acute stroke. J Cereb Blood Flow Metab. 1998;18(6):583-609.

5. Dijkhuizen RM, Nicolay K. Magnetic resonance imaging in experimental models of brain disorders. J Cereb Blood Flow Metab. 2003;23(12):1383-402.

6. Farr TD, Wegener S. Use of magnetic resonance imaging to predict outcome after stroke: a review of experimental and clinical evidence. J Cereb Blood Flow Metab. 2010;30(4):703-17.

7. Jiang Q, Zhang ZG, Chopp M. MRI of stroke recovery. Stroke. 2010;41(2):410-4.

8. Weber R, Ramos-Cabrer P, Hoehn M. Present status of magnetic resonance imaging and spectroscopy in animal stroke models. $\mathrm{J}$ Cereb Blood Flow Metab. 2006;26(5):591-604.

9. Calautti C, Baron JC. Functional neuroimaging studies of motor recovery after stroke in adults: a review. Stroke. 2003;34(6):155366.

10. Cramer SC, Bastings EP. Mapping clinically relevant plasticity after stroke. Neuropharmacology. 2000;39(5):842-51.

11. Van der Zijden JP, Dijkhuizen RM. Assessment of functional and neuroanatomical re-organization after experimental stroke using MRI. In: Ntziachristos V, Leroy-Willig A, Tavitian B, editors. Textbook of in vivo imaging in vertebrates. Chichester: Wiley; 2007. p. 239-41.

12. Assaf Y, Pasternak O. Diffusion tensor imaging (DTI)-based white matter mapping in brain research: a review. J Mol Neurosci. 2008;34(1):51-61.

13. Jiang Q, Zhang ZG, Chopp M. MRI evaluation of white matter recovery after brain injury. Stroke. 2010;41(10 Suppl):S112-3.

14. Mandeville JB, Rosen BR. Functional MRI. In: Toga AW, Mazziotta JC, editors. Brain mapping: the methods. 2nd ed. New York: Academic; 2002. p. 315-49.

15. Harel N, Ugurbil K, Uludag K, Yacoub E. Frontiers of brain mapping using MRI. J Magn Reson Imaging. 2006;23(6):945-57.

16. Masamoto K, Fukuda M, Vazquez A, Kim SG. Dose-dependent effect of isoflurane on neurovascular coupling in rat cerebral cortex. Eur J Neurosci. 2009;30(2):242-50.

17. Van der Linden A, Van Camp N, Ramos-Cabrer P, Hoehn M. Current status of functional MRI on small animals: application to physiology, pathophysiology, and cognition. NMR Biomed. 2007;20(5):522-45.

18. Rudin M, Mueggler T, Allegrini PR, Baumann D, Rausch M. Characterization of CNS disorders and evaluation of therapy using structural and functional MRI. Anal Bioanal Chem. 2003;377 (6):973-81.

19. Abo M, Chen Z, Lai LJ, Reese T, Bjelke B. Functional recovery after brain lesion-contralateral neuromodulation: an fMRI study. Neuroreport. 2001;12(7):1543-7.

20. Dijkhuizen RM, Ren J, Mandeville JB, Wu O, Ozdag FM, Moskowitz $\mathrm{MA}$, et al. Functional magnetic resonance imaging of reorganization in rat brain after stroke. Proc Natl Acad Sci U S A. 2001;98 (22):12766-71

21. Dijkhuizen RM, Singhal AB, Mandeville JB, Wu O, Halpern EF, Finklestein SP, et al. Correlation between brain reorganization, ischemic damage, and neurologic status after transient focal cerebral ischemia in rats: a functional magnetic resonance imaging study. J Neurosci. 2003;23(2):510-7.

22. Kim YR, van Meer MP, Mandeville JB, Tejima E, Dai G, Topalkara $\mathrm{K}$, et al. fMRI of delayed albumin treatment during stroke recovery in rats: implication for fast neuronal habituation in recovering brains. $\mathrm{J}$ Cereb Blood Flow Metab. 2007;27(1):142-53.

23. Kim YR, van Meer MP, Tejima E, Murata Y, Mandeville JB, Dai $\mathrm{G}$, et al. Functional MRI of delayed chronic lithium treatment in rat focal cerebral ischemia. Stroke. 2008;39(2):439-47.

24. Reese T, Porszasz R, Baumann D, Bochelen D, Boumezbeur F, McAllister KH, et al. Cytoprotection does not preserve brain functionality in rats during the acute post-stroke phase despite evidence of non-infarction provided by MRI. NMR Biomed. 2000;13(6):361-70.

25. Sauter A, Reese T, Porszasz R, Baumann D, Rausch M, Rudin M. Recovery of function in cytoprotected cerebral cortex in rat stroke model assessed by functional MRI. Magn Reson Med. 2002;47 (4):759-65.

26. Weber R, Ramos-Cabrer P, Justicia C, Wiedermann D, Strecker C, Sprenger $\mathrm{C}$, et al. Early prediction of functional recovery after experimental stroke: functional magnetic resonance imaging, electrophysiology, and behavioral testing in rats. J Neurosci. 2008;28(5):1022-9.

27. Sicard KM, Henninger N, Fisher M, Duong TQ, Ferris CF. Longterm changes of functional MRI-based brain function, behavioral status, and histopathology after transient focal cerebral ischemia in rats. Stroke. 2006;37(10):2593-600.

28. van Meer MP, van der Marel K, van der Sprenkel JW, Dijkhuizen RM. MRI of bilateral sensorimotor network activation in response to direct intracortical stimulation in rats after unilateral stroke. $\mathrm{J}$ Cereb Blood Flow Metab. 2011;31(7):1583-7.

29. Biswal B, Yetkin FZ, Haughton VM, Hyde JS. Functional connectivity in the motor cortex of resting human brain using echo-planar MRI. Magn Reson Med. 1995;34(4):537-41.

30. Auer DP. Spontaneous low-frequency blood oxygenation leveldependent fluctuations and functional connectivity analysis of the 'resting' brain. Magn Reson Imaging. 2008;26(7):1055-64.

31. Fox MD, Raichle ME. Spontaneous fluctuations in brain activity observed with functional magnetic resonance imaging. Nat Rev Neurosci. 2007;8(9):700-11.

32. van den Heuvel MP, van den Hulshoff Pol HE. Exploring the brain network: a review on resting-state fMRI functional connectivity. Eur Neuropsychopharmacol. 2010;20(8):519-34.

33. Honey CJ, Sporns O, Cammoun L, Gigandet X, Thiran JP, Meuli R, et al. Predicting human resting-state functional connectivity from structural connectivity. Proc Natl Acad Sci U S A. 2009;106(6):2035-40.

34. Vincent JL, Patel GH, Fox MD, Snyder AZ, Baker JT, Van Essen $\mathrm{DC}$, et al. Intrinsic functional architecture in the anaesthetized monkey brain. Nature. 2007;447(7140):83-6.

35. He BJ, Snyder AZ, Zempel JM, Smyth MD, Raichle ME. Electrophysiological correlates of the brain's intrinsic large-scale functional architecture. Proc Natl Acad Sci U S A. 2008;105(41):16039-44.

36. Laufs H, Krakow K, Sterzer P, Eger E, Beyerle A, Salek-Haddadi A, et al. Electroencephalographic signatures of attentional and cognitive default modes in spontaneous brain activity fluctuations at rest. Proc Natl Acad Sci U S A. 2003;100(19):11053-8.

37. Lu H, Zuo Y, Gu H, Waltz JA, Zhan W, Scholl CA, et al. Synchronized delta oscillations correlate with the resting-state functional MRI signal. Proc Natl Acad Sci U S A. 2007;104(46):18265-9.

38. Kannurpatti SS, Biswal BB, Kim YR, Rosen BR. Spatio-temporal characteristics of low-frequency BOLD signal fluctuations in isoflurane-anesthetized rat brain. NeuroImage. 2008;40(4):1738-47. 
39. Majeed W, Magnuson M, Keilholz SD. Spatiotemporal dynamics of low frequency fluctuations in BOLD fMRI of the rat. J Magn Reson Imaging. 2009;30(2):384-93.

40. Pawela CP, Biswal BB, Cho YR, Kao DS, Li R, Jones SR, et al. Resting-state functional connectivity of the rat brain. Magn Reson Med. 2008;59(5):1021-9.

41. Wang K, van Meer MP, van der Marel K, van der Toorn A, Xu L, Liu Y, et al. Temporal scaling properties and spatial synchronization of spontaneous blood oxygenation level-dependent (BOLD) signal fluctuations in rat sensorimotor network at different levels of isoflurane anesthesia. NMR Biomed. 2011;24(1):61-7.

42. Zhao F, Zhao T, Zhou L, Wu Q, Hu X. BOLD study of stimulation-induced neural activity and resting-state connectivity in medetomidine-sedated rat. NeuroImage. 2008;39(1):248-60.

43. van Meer MP, van der Marel K, Otte WM, Berkelbach van der Sprenkel JW, Dijkhuizen RM. Correspondence between altered functional and structural connectivity in the contralesional sensorimotor cortex after unilateral stroke in rats: a combined restingstate functional MRI and manganese-enhanced MRI study. J Cereb Blood Flow Metab. 2010;30(10):1707-11.

44. van Meer MP, van der Marel K, Wang K, Otte WM, El Bouazati S, Roeling TA, et al. Recovery of sensorimotor function after experimental stroke correlates with restoration of resting-state interhemispheric functional connectivity. J Neurosci. 2010;30(11):3964-72.

45. Carter AR, Astafiev SV, Lang CE, Connor LT, Rengachary J, Strube MJ, et al. Resting interhemispheric functional magnetic resonance imaging connectivity predicts performance after stroke. Ann Neurol. 2010;67(3):365-75.

46. Silva AC, Lee JH, Aoki I, Koretsky AP. Manganese-enhanced magnetic resonance imaging (MEMRI): methodological and practical considerations. NMR Biomed. 2004;17(8):532-43.

47. Pautler RG, Silva AC, Koretsky AP. In vivo neuronal tract tracing using manganese-enhanced magnetic resonance imaging. Magn Reson Med. 1998;40(5):740-8.

48. Bullmore E, Sporns O. Complex brain networks: graph theoretical analysis of structural and functional systems. Nat Rev Neurosci. 2009;10(3):186-98

49. Reijneveld JC, Ponten SC, Berendse HW, Stam CJ. The application of graph theoretical analysis to complex networks in the brain. Clin Neurophysiol. 2007;118(11):2317-31.

50. Watts DJ, Strogatz SH. Collective dynamics of 'small-world' networks. Nature. 1998;393(6684):440-2.

51. Wang L, Yu C, Chen H, Qin W, He Y, Fan F, et al. Dynamic functional reorganization of the motor execution network after stroke. Brain. 2010;133(Pt 4):1224-38.

52. Wise RG, Tracey I. The role of fMRI in drug discovery. J Magn Reson Imaging. 2006;23(6):862-76.

53. Reese T, Bochelen D, Baumann D, Rausch M, Sauter A, Rudin M. Impaired functionality of reperfused brain tissue following short transient focal ischemia in rats. Magn Reson Imaging. 2002;20 (6): $447-54$

54. Hoff EI, Steinbusch HW, van Oostenbrugge RJ, Garrett L, Otte WM, van der Marel K, et al. Alterations in the cholinergic system after frontal cortical infarction in rat brain: pharmacological magnetic resonance imaging of muscarinic receptor responsiveness and stereological analysis of cholinergic forebrain neurons. Neurobiol Dis. 2011;43(3):625-34.

55. Basser PJ, Jones DK. Diffusion-tensor MRI: theory, experimental design and data analysis - a technical review. NMR Biomed. 2002;15(7-8):456-67.

56. Mori S, Zhang J. Principles of diffusion tensor imaging and its applications to basic neuroscience research. Neuron. 2006;51 (5):527-39.

57. Sotak $\mathrm{CH}$. The role of diffusion tensor imaging in the evaluation of ischemic brain injury - a review. NMR Biomed. 2002;15(78):561-9.

58. van der Zijden JP, van der Toorn A, van der Marel K, Dijkhuizen RM. Longitudinal in vivo MRI of alterations in perilesional tissue after transient ischemic stroke in rats. Exp Neurol. 2008;212 (1):207-12.

59. Liu Y, D'Arceuil HE, Westmoreland S, He J, Duggan M, Gonzalez $\mathrm{RG}$, et al. Serial diffusion tensor MRI after transient and permanent cerebral ischemia in nonhuman primates. Stroke. 2007;38(1):13845.

60. Jiang Q, Zhang ZG, Ding GL, Silver B, Zhang L, Meng H, et al. MRI detects white matter reorganization after neural progenitor cell treatment of stroke. NeuroImage. 2006;32(3):1080-9.

61. Ding G, Jiang Q, Li L, Zhang L, Zhang ZG, Ledbetter KA, et al. Magnetic resonance imaging investigation of axonal remodeling and angiogenesis after embolic stroke in sildenafil-treated rats. $\mathrm{J}$ Cereb Blood Flow Metab. 2008;28(8):1440-8.

62. Li L, Jiang Q, Ding G, Zhang L, Zhang ZG, Li Q, et al. MRI identification of white matter reorganization enhanced by erythropoietin treatment in a rat model of focal ischemia. Stroke. 2009;40(3):936-41.

63. Schaechter JD, Fricker ZP, Perdue KL, Helmer KG, Vangel MG, Greve DN, et al. Microstructural status of ipsilesional and contralesional corticospinal tract correlates with motor skill in chronic stroke patients. Hum Brain Mapp. 2009;30(11):3461-74.

64. Zhan W, Yang Y. How accurately can the diffusion profiles indicate multiple fiber orientations? A study on general fiber crossings in diffusion MRI. J Magn Reson. 2006;183(2):193-202.

65. Jensen JH, Helpern JA, Ramani A, Lu H, Kaczynski K. Diffusional kurtosis imaging: the quantification of non-Gaussian water diffusion by means of magnetic resonance imaging. Magn Reson Med. 2005;53(6):1432-40.

66. Tuch DS, Reese TG, Wiegell MR, Wedeen VJ. Diffusion MRI of complex neural architecture. Neuron. 2003;40(5):885-95.

67. Tournier JD, Calamante F, Gadian DG, Connelly A. Direct estimation of the fiber orientation density function from diffusionweighted MRI data using spherical deconvolution. NeuroImage. 2004;23(3):1176-85.

68. Wedeen VJ, Wang RP, Schmahmann JD, Benner T, Tseng WY, Dai $\mathrm{G}$, et al. Diffusion spectrum magnetic resonance imaging (DSI) tractography of crossing fibers. NeuroImage. 2008;41(4):1267-77.

69. Jensen JH, Helpern JA. MRI quantification of non-Gaussian water diffusion by kurtosis analysis. NMR Biomed. 2010;23(7):698-710.

70. Wu EX, Cheung MM. MR diffusion kurtosis imaging for neural tissue characterization. NMR Biomed. 2010;23(7):836-48. 